\title{
Hacia un ontológico de Creatividad aplicado al contexto del Diseño y Artesanía: Innovación y Computación Evolutiva.
}

Cayetano José Cruz García / Alfonso González González² / Francisco Fernández de Vega ${ }^{3}$ Universidad de Extremadura

\section{Resumen}

La Artesanía es un medio de producción importante, que aporta valores socio-culturales y turísticos que necesitan de una adaptación a la sociedad contemporánea, en la que la convivencia con la innovación tecnológica puede ser más activa y provechosa. En el presente artículo se discierne sobre un estado de intenciones, dirigido a un ontológico particular para el Diseño y la Artesanía, donde la acción creativa de "caja negra" del individuo se materialice en un método visible. La particularidad artesanal necesita de modelos singulares en pro de la innovación, cada vez más posibles, y que precisan de: aprender de los procesos creativos del pensamiento creativo del individuo, elaborar un método ontológico de diseño específico para un escenario particular, y analizar y aplicar las relaciones entre la Tecnología/Inteligencia Artificial y la Artesanía, como dos mundos inicialmente dispares en el que encontrar relaciones de sinergia.

Palabras clave: Artesanía, computación, diseño de producto, metodología, algoritmos evolutivos.

\section{Towards an ontological of Creativity applied to the context of Design and Crafts: Innovation and Evolutionary Computing}

\begin{abstract}
The Handicrafts sector is an important means of production, which provides socio-cultural and tourist values that can and need to be adapted to contemporary society, in which coexistence with technological innovation can be more active and profitable. The present article discerns a state of intentions, addressed to a particular ontology for Design and Craftsmanship, where the creative action of the individual's "black box" is materialized in a visible method. The peculiarity of these sectors requires singular models in favour of innovation, more and more possible, which require: learning from the creative processes of the creative thought of the individual, elaborating an ontological method of specific design for a particular scenario, and analysing and applying the relations between Technology/Artificial Intelligence and Handicrafts, as two initially disparate worlds in which to find relations of synergy. the system. The proposed model reflects a modular structure that can be adapted to the particularities and characteristics of each project, aiming to generate a broad view of Sociotechnical Innovations.
\end{abstract}

Keywords: Craft, computer, product design, methodology, evolutionary algorithms.

\footnotetext{
PhD. Cayetano José Cruz García. Área de Dibujo. Centro Universitario de Mérida. Universidad de Extremadura. email: ccruz@unex.es

2 PhD. Alfonso González González. Área de Procesos de Fabricación. Centro Universitario de Mérida Universidad de Extremadura. email: agg@unex.es

3 PhD.Francisco Fernández de Vega. Área de Arquitectura y Tecnología de Computadores. Universidad de Extremadura. email: fcofdez@unex.es
} 
Los actuales procesos metodológicos en diseño están en continuo progreso, y establecen la necesidad de generar nuevos escenario ${ }^{4}$ aplicados a contextos específicos y a empresas, cuyas particularidades pasan por la necesidad de atender y controlar todos los procesos que influyen en su cadena de valor, como puede ser el caso de las microempresas productoras. Una casuística concreta la tenemos en las empresas artesanas, que viven una problemática específica:Tienen que competir con la globalización sin perder sus rasgos particulares, de corta producción o única, venta directa y/o relación directa con los usuarios, participación humana en los procesos de producción, sello de autor,..., ${ }^{5}$ y que también están vinculados a las características de una micro empresa ${ }^{6}$. Por lo que, si éstas son sus premisas, se necesitan soluciones que arropen a la artesanía y estén en consonancia con su condición.

En años precedentes se han aplicado acciones de éxito en la empresa artesana, que han permitido incorporar factores de innovación y experiencias desde su vinculación a Diseñadores de Producto, fruto de un plan estratégico nacional ${ }^{7}$. Estas acciones han sido aplicadas en las diversas Comunidades españolas, a partir de unas líneas estratégicas que fueron planificadas desde el Observatorio Nacional de Artesanía, y que han dado como resultado mejoras en el sector; tales como son las actuaciones D'Artes ${ }^{8}$, y otras motivadas desde diferentes instituciones, como la Universidad de Extremadura en su vínculo con diseñadores industriales ${ }^{9}$. El sector artesano es un medio de producción importante, que aporta valores socio-culturales y turísticos que pueden y necesitan de una adaptación a la sociedad contemporánea, en la que la convivencia con la innovación tecnológica puede ser más activa y provechosa.

\footnotetext{
4 Martínez Torán, M., et al. (2012) Escenarios de futuro de la artesanía española: método de estudio. DEFORMA Cultura Online: http://www.deforma.info/es/product.php?id_product=66

5 Martínez Torán, M. (2015) Nuevas actitudes y modelos de negocio entre diseño y artesanía. MasD.

6 Martínez Torán, M., Esteve Sendra, C. and Moreno Cuesta, R. (2017) Scenarios for design and craft. The Design Journal 20.sup1: S2778-S2788.

7 Varios (2006) Recomendaciones para la promoción de la Artesanía en España. Ministerio de Industria Turismo y Comercio: Oficio y Arte.

8 https://ecitydoc.com/download/dartes-ii-diseno-y-artesania-en-extremadura_pdf\#

9 Cruz, C. (2012) Corporeidad de una experiencia metodológica - diseño industrial CUM - Terracota Mérida. Oficio y Arte, 122, p.14.
} 
La Artesanía tiene entre sus grandes retos adaptarse a los nuevos tiempos sin perder sus rasgos diferenciales ni desaparecer. Por lo general, una empresa artesana tiene dificultades para posicionarse y competir, en la gestión de procesos de producción y distribución, y en relación con la atención personalizada en la comercialización de sus productos ${ }^{10}$.

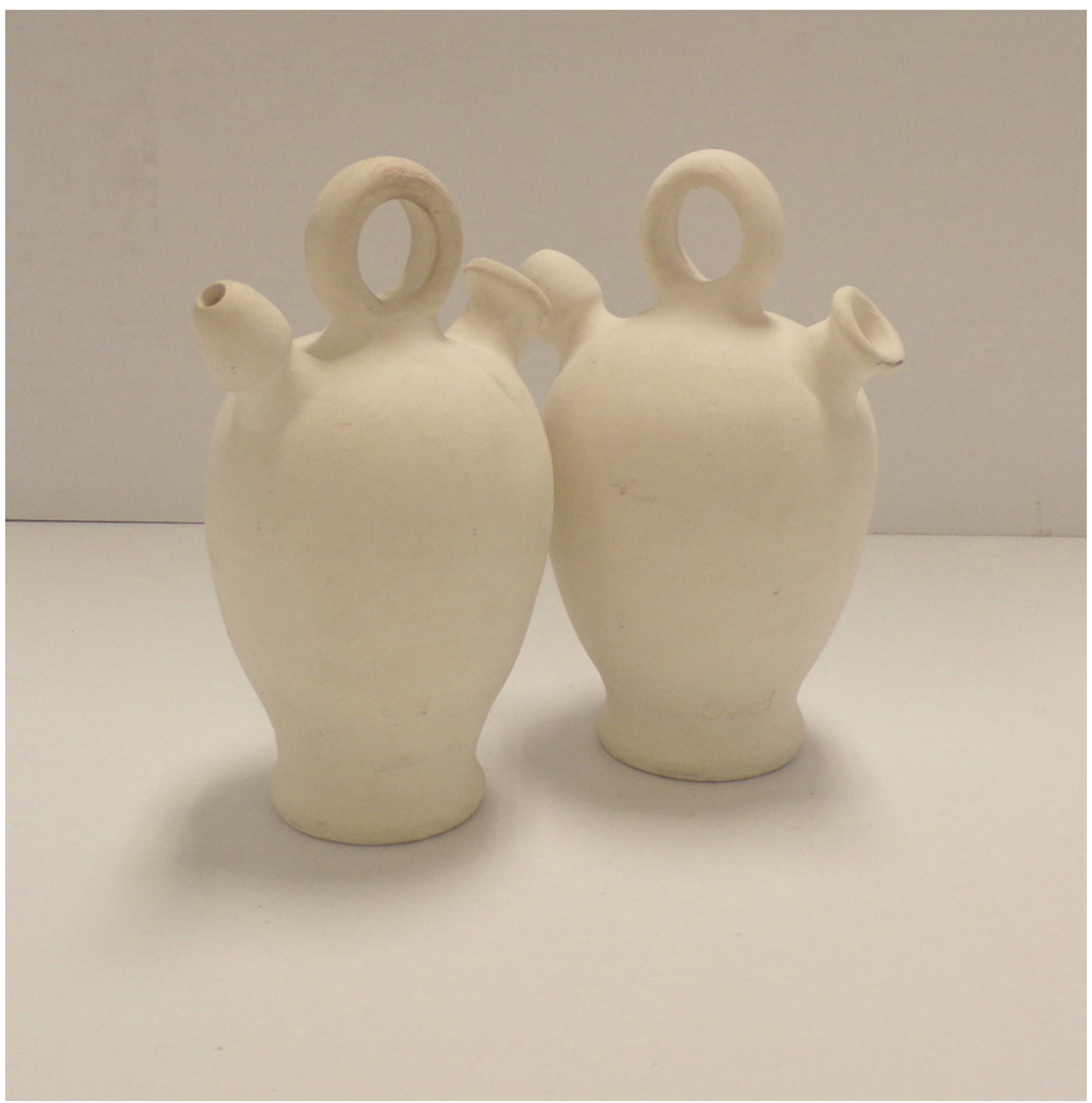

Fig. 1. CJC, AGG. Botijos realizados en torno. Solución con leves variaciones en la seriación, realizados por Terracota Mérida (España)

10 Nombela, G. (coord.) (2010). Ideas para una nueva economía: hacia una España más sostenible en 2025. Madrid: Fundación IDEAS. 


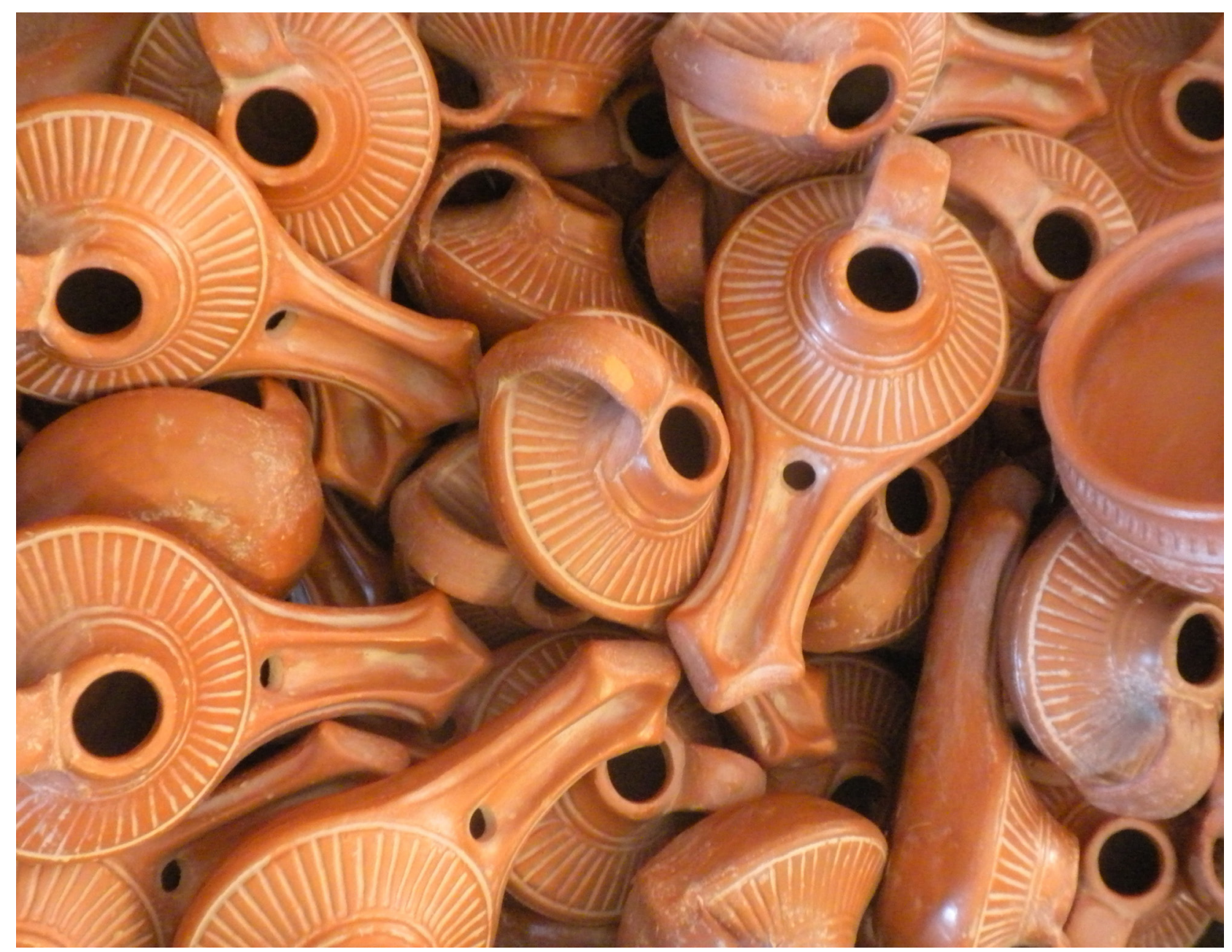

Fig. 2. CJC, AGG Reproducciones en serie de lucernas romanas, realizadas por Terracota Mérida (España).

En cuanto a las peculiaridades del sector, una de las facetas más evidentes de la Artesanía es la limitada producción, que va desde la elaboración de piezas únicas (propias de la Artesanía Artística) (fig. 1) a la corta tirada de piezas seriadas (fig. 2). Los procesos de producción dotan al resultado de rasgos formales diferenciados entre los productos, evidentes en mayor o menor medida, y la acción del hacedor (artesano) está presente, al menos, en el proceso de fabricación. Igualmente, los mecanismos de venta directa y de relación en el comercio habitual coinciden con la necesidad de interactuar en la estructura hacedor - producto - usuario ${ }^{11}$. Sin embargo, este paradigma es diferente al del Artista, que visualiza la producción como un medio del pensamiento, pero que también puede tener confluencias con la producción artesana. Tanto la actividad "hacedora" como la del "pensamiento", pueden encontrar confluencias con los avances de la tecnología y la computación, incluyendo la posible aplicabilidad de la inteligencia artificial (IA). Así, encontramos los siguientes rasgos coincidentes con las dinámicas propias de la Artesanía y la creatividad computacional, tales como la capacidad de producir un ilimitado número de soluciones diferentes bajo unos mismos parámetros, la relación de interacción hacedor (sea hombre o máquina) - proceso (sea tecnología directa o artesanal) - Usuario 
(interacción), o la preocupación por hacer valer la evidencia creativa humana. Esta casuística entronca con la visibilidad tan opuesta que parecen tener ambos mundos, y que parecen converger conforme evoluciona el mundo contemporáneo.

\section{Hacia un ontológico creativo para escenarios particulares}

La creatividad es una faceta compleja de nuestro pensamiento, y la acción creativa se pone de manifiesto en disciplinas concretas del quehacer humano ${ }^{12}$. Para el diseñador se visualiza desde una metodología, clave a la hora de obtener nuevas soluciones; que en la acción proyectual se manifiesta en la capacidad de idear, imaginar, intuir, ... El diseñador elabora propuestas bajo el amparo de un método, que le diferencia en la manera de resolver problemas o proponer soluciones ${ }^{13}$. Este método responde a una serie de técnicas metodológicas implementadas en un proceso de diseño (ontológico) ${ }^{14}{ }^{15}$, en el que se dispone un recorrido de actuación. Sin embargo, la inteligencia creativa de este diseñador debiera ser lo más cualificada posible, para que la aportación de su pensamiento actuara en el proceso con más determinación ${ }^{16}$.

El progreso y la sabiduría proyectual requieren, entonces, de una ejercitación para habilitar la capacidad intuitiva personal en el quehacer creativo, lo que se describe como "caja negra"17, frente a los procesos más habituales de "caja trasparente". Por lo general, los ontológicos de diseño incorporan alguna fase donde se inspire la acción creativa (de caja negra) durante su progreso metodológico. Sin embargo, el proceso puede llevar a soluciones en el que no se manifieste tal pensamiento creativo. Comprender esta circunstancia es fundamental, porque a veces el diseñador se ve envuelto en automatismos que pueden deshabilitar el pensamiento divergente o el flujo creativo $^{18}$, por eso es fundamental un adiestramiento creativo ${ }^{19}$. Los artistas artesanos, por lo contrario, generalmente disponen de habilidad creativa, pero no de método. Ante el reto de hacer cohesionar ambos procesos, investigadores como J. Albers elaboraron ejercitaciones de desarrollo secuencial ${ }^{20}$, tanto para adiestrar la capacidad del ser creativo como su ejercitación metodológica.

El conjunto de ejercitaciones que estimulan y forman para capacitar en la creatividad, es lo que se denomina creática ${ }^{21}$. En este sentido, la acción de formar un imaginario nuevo puede establecerse desde diferentes métodos $^{22}$, pero dichas metodologías manifiestan la creatividad a través de Técnicas metodológicas (brainstorming,.. ) y no como un proceso.

\footnotetext{
12 Sawyer, R. K. (2011). Explaining creativity: The science of human innovation. Oxford University Press.

13 Cross, N. (2006). Formas de Conocimiento. Londres: Springer

14 Jones, J. C. (1984). Un método de diseño sistemático, en N. Cross (Ed.), Developments in Design Methodology (pp. 9-31). Chichester: John Wiley \& Sons

15 Green, S., Southee, D., \& Boult, J. (2014). Towards a design process ontology. The Design Journal, 17(4), 515-537.

16 Cropley, D. H. (2015). Promoting creativity and innovation in engineering education. Psychology of Aesthetics, Creativity, and the Arts, 9(2), 161-171.

17 Guilford, J. P. (1950). Creativity. American Psychologist. 5, 444-454.

18 Csikszentmihalyi, Mihaly. (1996). Creativity: Flow and the Psychology of Discovery and Invention. New York: Harper Collins Publishers

19 Cruz García, Cayetano José. (2013). Idear la forma: Capacitación creativa. Cuadernos del Centro de Estudios en Diseño y Comunicación. Ensayos, 43: 113-125.

20 Dantzic, C. M. (1999). How to draw. A complete guide to techniques and appreciation. London: Laurence King.

21 De la Torre, S. (1995). Creatividad aplicada. Recursos para una formación creativa. Madrid: Escuela Española.

22 Purcell, A., \& Gero, J. S. (1998). Drawings and the design process: A review of protocol studies in design and other disciplines and related research in cognitive psychology. Design Studies 19(4), 389-430.
} 
El presente planteamiento toma como argumento la ejercitación secuencial y generativa para elaborar un futuro método, que parte de la experiencia obtenida de investigaciones anteriores: Una como elaboración de un modelo de dibujo basado en principios secuenciales, sostenido bajo normas hápticas ${ }^{23}$, motivado por el interés en adiestrar para idear la forma desde la capacitación creativa personal. La acción secuencial, se realiza tanto en el reconocimiento háptico ${ }^{24}$, como en uno de sus principios fundamentales, como el Principio de Percepción Sucesiva ${ }^{25}$. Otra a partir de investigaciones desde la perspectiva de los Algoritmos Evolutivos (EAs), que analizan el comportamiento de los artistas cuando emulan a la computadora; lo que se denomina como Algoritmos Evolutivos Desconectados (UEAs) 2627.

Estas dos líneas de investigación indicadas anteriormente establecen que una ejercitación en procesos secuenciales permite: tanto la comprensión de la realidad de un objeto, como la posible elaboración de una propuesta imaginada, y cuyo argumento está en el propio proceso. De tal manera, se podría establecer un ontológico que tenga como sustento el proceso secuencial y generativo ${ }^{28}$, y elevarlo a diferentes fases y necesidades a las que se enfrenta una empresa artesana. Por ejemplo, si nos situamos en una fase de logística, y una empresa artesana cuenta con una variedad amplia de piezas únicas o de piezas de seriación corta, puede necesitar un embalaje único que se adapte a la mayor parte de peculiaridades de todos sus productos. Por lo tanto, el embalaje pudiera diseñarse en base a unos parámetros, que un algoritmo evolutivo adapte a las características que el producto sugiere; igualmente, un posible comprador podría participar en la toma de decisiones, frente a todas las propuestas que establezca un algoritmo evolutivo. De esta manera, el artesano potencia rasgos propios tanto de la cualidad de su sector (personalidad, diferenciación) como mecanismos de comercialización en los que la venta directa está presente.

El ejemplo anterior indica cómo un ontológico particular participa en el proceso de diseño, manteniendo los principios propios de la creatividad "hacedora" que están presentes en el pensamiento del "hacedor". El "pensamiento creativo" se identifica en el proceso de actuación del artesano.

Investigaciones recientes ponen en consideración cómo los objetos inspiran o comunican sus propios valores, de tal manera que los objetos bien diseñados nos hablan de para qué fueron concebidos, sus cualidades, cómo pueden ser usados, etc. Este discurso sobre la relación de interacción y comunicación es lo que se denomina teoría de affordances ${ }^{29}{ }^{30}$. La artesanía tradicional y la evolución del arte son la respuesta a una evolución sosegada, en la que los procesos de optimización y verificación han sido madurados y verificados lentamente, hasta lograr un affordances del producto adecuado. Por lo tanto, incorporar este mismo ontológico

\footnotetext{
23 Cruz García, Cayetano José. (2006). Un modelo háptico de dibujo artístico para diseño industrial. Metodología para la ideación y creatividad. Actas de Diseño, 1: $215-216$.

24 Hippius, R (1934). Erkennendes Tasten, Neue Psychologische Studien, 10.

25 Revest (1950). Psychology and art of the blind. London: Longmans Green\&Co.

26 Fernández de Vega, F., Navarro, L., Cruz, C., Chavez, F., Espada, L., Hernandez, P., Gallego, T (2013) Unplugging evolutionary algorithms: on the sources of novelty and creativity. Evolutionary Computation (CEC), 2013 IEEE Congress on, pp. 2856-2863. IEEE.

27 Cruz, C., Fernández de Vega, F, Hernández, P., Navarro, L., Gallego, T., Espada, L.: Arte (2015). Arte Evolutivo y Computación: Diálogos de un experimento. Cáceres: Universidad de Extremadura.

28 Prats, M., Earl, C., Garner, S., \& Jowers, I. (2006). Shape exploration of designs in a style: toward generation of product designs. Artificial Intelligence for Engineering Design, Analysis and Manufacturing 20(3), 201-215.

29 Gibson, J. J. (1977). The Theory of Affordances, in Robert E. Shaw \& John Bransford (Eds.). Perceiving, Acting, and Knowing. Hillsdale, NJ: Lawrence Erlbaum Associates.

30 Norman, D. A. (1999). Affordance, conventions, and design. Interactions 6(3), 38-43.
} 
a procesos de diseño y a la capacidad tecnológica y computacional sería coherente en este contexto y sus posibles escenarios, en los que la creatividad se manifieste para con los intereses de la Artesanía, el Diseño o el Arte.

\section{Paramétrica y Algoritmos creativos. Sinergias en el Ontológico particular}

La creatividad computacional ha cobrado impulso en la última década. Mientras que la música y el arte eran los principales temas tratados en los años 70 (cuando se mostraron los primeros intentos de dotar a los ordenadores de capacidades creativas, véase la exposición Serendipity, Londres) ${ }^{31}$, hoy en día se abordan muchas áreas diferentes: en arte ${ }^{32}$; de la narrativa a los juegos; de la música a la animación; de los cuentos originales a la poesía; de la curiosidad computacional a la danza robótica; del diseño de sabores a la improvisación jazzística. Por lo tanto, hay especial interés y se necesitan nuevos medios para generar contenidos creativos que puedan adaptarse dinámicamente a diferentes contextos ${ }^{33}$. En este sentido, en los últimos años se ha desarrollado un nuevo modelo de creación artística por ordenador, con el objetivo principal de insertar artistas humanos en el núcleo del algoritmo: la versión desconectada del algoritmo evolutivo ${ }^{34}$. No sólo se producía trabajo artístico mediante la aplicación de operaciones genéticas por parte de artistas humanos, sino que se consideraba un análisis actualizado de los principales actores del mundo del arte: galerías y museos, público asistente a exposiciones y también críticos de arte ${ }^{35}$. Podemos encontrar una plétora de algoritmos que producen arte $^{36}$, pero no muchos de los autores detrás de los algoritmos han intentado exhibir el trabajo en galerías de arte o museos. Por otro lado, cuando el algoritmo creativo es autónomo, una aplicación adecuada de los principios de diversidad disponibles en los algoritmos evolutivos sería útil para el diseño en masa y la producción de artefactos únicos. En lugar de centrarnos en las capacidades de "explotación" del algoritmo al converger hacia una única solución, podríamos explotar mejor sus capacidades de "exploración" que conducen a la diversidad ${ }^{37}$, que combinadas con poblaciones masivas y sistemas informáticos paralelos y distribuidos, podrían utilizarse para diseñar series de productos con un diseño único, es decir, se pueden producir miles de instancias del mismo producto con características individuales. Aunque se ha buscado la diversidad en algunos trabajos anteriores, las poblaciones masivas y sus capacidades inherentes nunca han sido consideradas en este contexto.

En el caso de la fabricación de productos, los contenidos pueden ser elaborados desde lo bidimensional a lo tridimensional, lo que inicialmente conlleva un proceso gráfico similar al aplicado en la Creatividad Computacional, pero cuyo pensamiento debe soportar una serie de parámetros cuya abstracción tendría que

\footnotetext{
31 Usselmann, R. (2013) The dilemma of media art: cybernetic serendipity at the ICA London, Leonardo 36(5):389-396.

32 Lutton, E. (2006) Evolution of fractal shapes for artists and designers", International Journal on Artificial Intelligence Tools 15(4):651-672.

33 "IEEE Computational Inelligence Society, Task Force on Creative Intelligence" http://cilab.cs.ccu.edu.tw/ci-tf/

34 Fernández de Vega, F., Cruz, C., Navarro, L., Hernández, P., Gallego, T. \& Espada, L. (2014) Unplugging Evolutionary Algorithms: an experiment on human-algorithmic creativity Genet. Program. Evol. Machines 15(4):379-402

35 Navarro, L., Fernández de Vega, P., Hernández, P., \& Cruz C. (2016) Analysing creative models based on unplugged evolutionary algorithms. IEEE Congress on Evolutionary Computation - CEC, pp. 4570-4577

36 McCormack, J. \& D'Inverno, M. (2012) Computers and creativity: The road ahead. Computers and creativity, pp. $421-424$.

37 Ursem, R. K.(2002) Diversity-guided evolutionary algorithms. International Conference on Parallel Problem Solving from Nature PPSN, pp. 462-471, Springer Berlin Heidelberg
} 
responder a condiciones de valor del producto ${ }^{38}$ (fig. 3). Por lo tanto, lo importante es que en el ontológico del método el proceso forme parte de la confección del producto. El proceso creativo que puede dar lugar a una obra de arte ${ }^{39}$, sin duda, comparte mucho en común con el proceso de diseño, y cuyas habilidades pueden ser necesarias para ambos ${ }^{40}$.

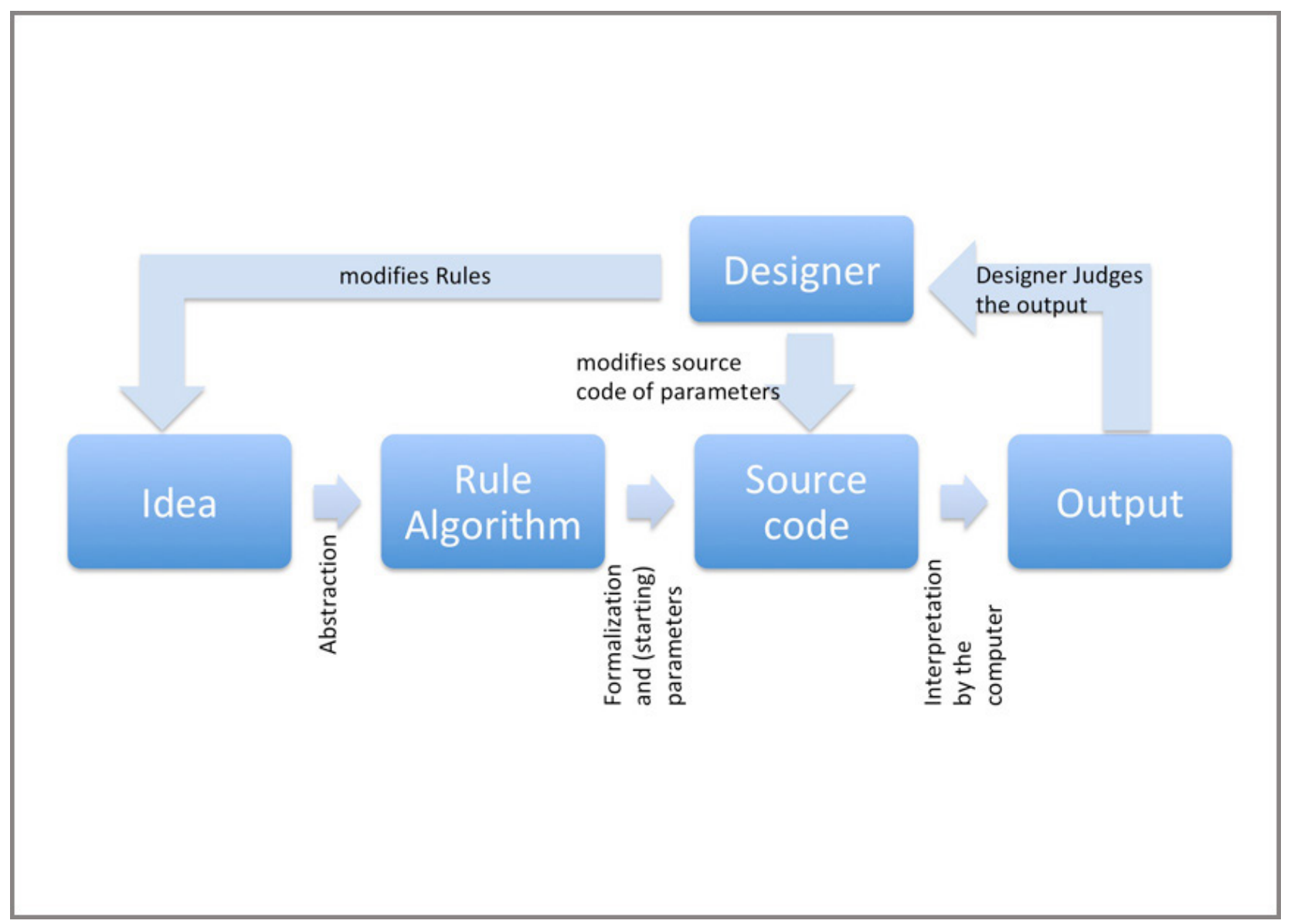

Fig. 3. AGG. Mapa proceso atendiendo a lo establecido por los autores en Generative Gestaltung. Copyright Groß, B., Laub, J. and Lazzeroni, Cl. (2009).

Al igual que un diseñador de producto establece parámetros en base a una estructura morfológica de Layout $^{41}$, donde el dibujo es un elemento esencial ${ }^{42}$ y que se implementa desde la fase de ideación al ComputerAided Design $(\mathrm{CAD})^{43}$, un Artesano pudiera identificar e implementar un Lay-out en un proceso de Computer-

38 Groß, B., Laub, J. and Lazzeroni, Cl. (2009). Generative Gestaltung. Entwerfen, Programmieren, Visualisieren mit Processing. Mainz: Verlag Hermann Schmidt.

39 Bohnacker, H., Gross, B., Laub, J., \& Lazzeroni, C. (2012). Generative design: visualize, program, and create with processing. Princeton Architectural Press.

40 Lawson, B. (2006). How designers think: the design process demystified. Routledge.

41 Karjalainen, T-M. (2007). It Looks Like a Toyota: Educational Approaches to Designing for Visual Brand Recognition. International Journal of Design 1, 1, pp. 67-81.

42 Tovey, M. (1989). Drawing and CAD in industrial design. Design Studies, 10(1), 24-39.

43 Kelley, D. S., Newcomer, J. L., \& McKell, E. K. (2001). The Design Process Ideation and Computer-Aided Design. ASEE Annual 
Aided Craftsmanship (CAC), para asistir a la artesanía en los procesos de ideación, control metodológico o productos asociados a necesidades asociadas a la pieza artesana; como por ejemplo un embalaje adaptable, que puede hacer uso de la fabricación integrada por ordenador (CIM $)^{44}$, tal y como se hace en los sistemas modernos de fabricación y transformación del diseño del producto ${ }^{45} 46$.

El objetivo de la planificación de procesos, por un lado, es determinar los recursos de fabricación y las secuencias de operaciones apropiadas para cada trabajo en el sistema. La información que sintetiza un Layout, proporciona la información necesaria sobre los procesos de fabricación y sus parámetros ${ }^{47} 48$.

La planificación y programación integrada de procesos, tiene correspondencia con la creatividad y atiende a los problemas combinatorios difíciles, que se desarrollan desde el ontológico del proceso ${ }^{49}$. Por lo tanto, la función de pensamiento que una computadora puede atender requiere de una parametrización, que puede tener presente aquellas señas de identidad que el artesano quiera hacer prevalecer. Se trata de que la Artesanía potencie sus valores y la tecnología se adapte a sus necesidades.

Los algoritmos evolutivos (EA) son uno de los más famosos algoritmos metaheurísticos basados en los principios de la evolución darwiniana que encontraron su uso en diversas ramas de la ciencia. Los EA modernos demostraron ser muy confiables en la búsqueda de planes y procesos creativos ${ }^{50}$. En esta línea, se pretende dar concreción a soluciones que implementen soluciones particulares, mediante la implementación de algoritmos evolutivos que permitan implementar el signo creativo, imbuidos en los procesos de diseño y fabricación de productos artesanos, y la interacción hombre - máquina ${ }^{51}$. (fig. 4). (fig. 5)

\footnotetext{
Conference Exposition, 5, 1.
}

44 Kim, K.H. \& Egbelu, P. J. (1999). Scheduling in a production environment with multiple process plans per job, International Journal of Production Research, Vol. 37, No. 12, pp. 2725-2753.

45 Li, X., Gao, L., Zhang, C., \& Shao, X. (2010). A review on Integrated Process Planning and Scheduling, International Journal of Manufacturing Research, Vol. 5, No. 2, 161-180.

46 Shao, X., Li, X, Gao, L. \& Zhang, C., (2009). Integration of process planning and scheduling - A modified genetic algorithmbased approach, Computers \& Operations Research, Vol. 36, No. 6, pp. 2082-2096.

47 Li, X., Wen, X. \& Gao, L. (2014). An Effective Genetic Algorithm for Multi-objective Integrated Process Planning and Scheduling with Various Flexibilities in Process Planning, Journal of Universal Computer Science, Vol. 20 No. 14, pp. 1926-1950.

48 Amin-Naseri, M. R. \& Afshari, A.J. (2012). A hybrid genetic algorithm for integrated process planning and scheduling problem with precedence constraints, International Journal of Advanced Manufacturing Technology, Vol. 59, No. 1, pp. $273-287$.

49 Fernández, M., Gómez-Pérez, A., \& Juristo, N. (1997). METHONTOLOGY: from ontological art towards ontological engineering. In Proceedings of the Ontological Engineering AAAl-97 Spring Symposium Series (pp. 33-40). Stanford, CA.

50 Prats, M., Earl, C., Garner, S., \& Jowers, I. (2006). Shape exploration of designs in a style: toward generation of product designs. Artificial Intelligence for Engineering Design, Analysis and Manufacturing 20(3), 201-215.

51 Dix, A. (2004) Human computer interaction. Pearson Education. 


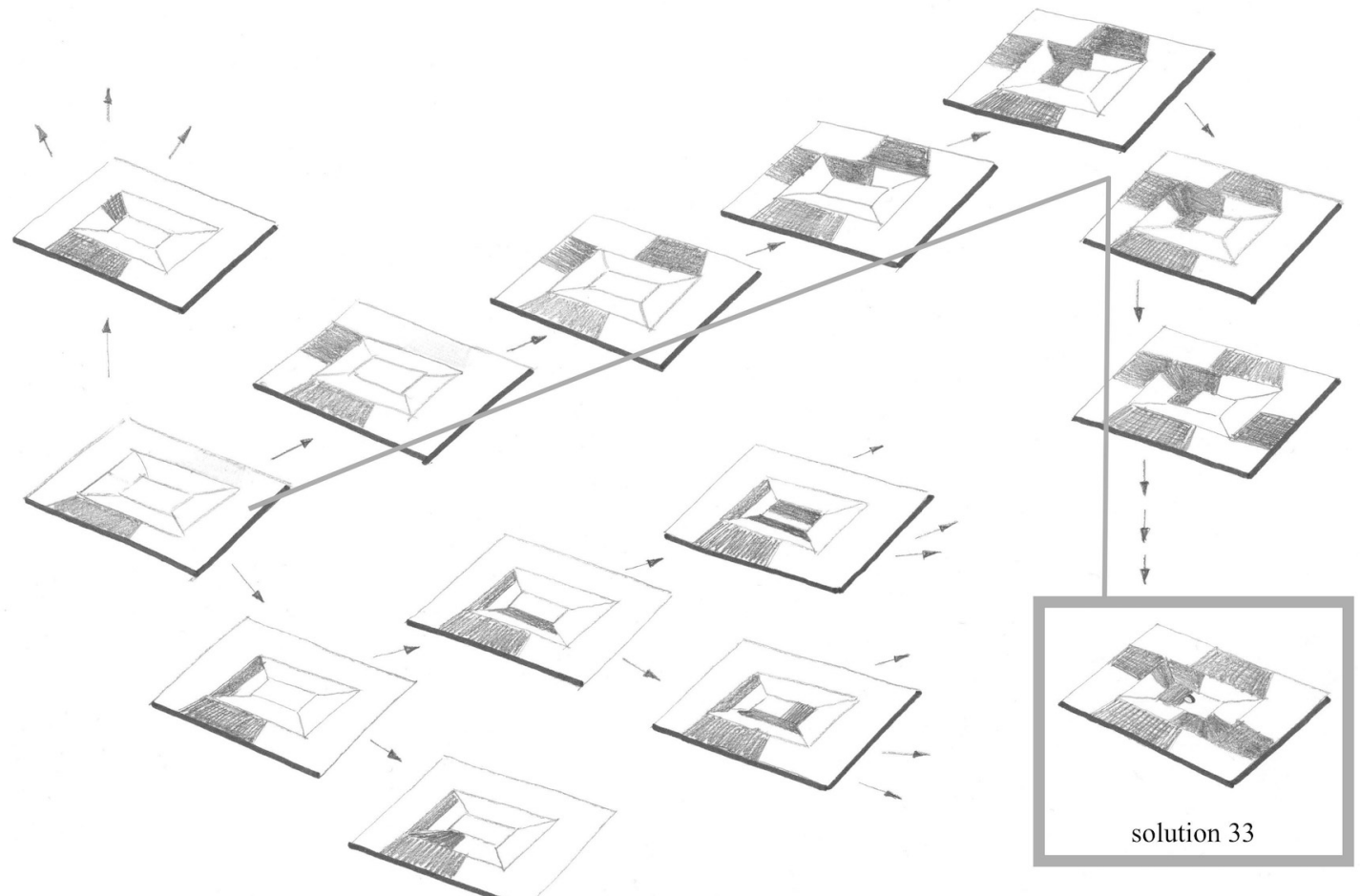

Fig. 4. CJC. Solución de desarrollo evolutivo. Propuesta de Diseño ornamental para lavabo Modelo Caja. Solución generada evolutivamente. Diseñado por Cayetano Cruz para Artesanía del Vidrio, Andrea Seimandi.
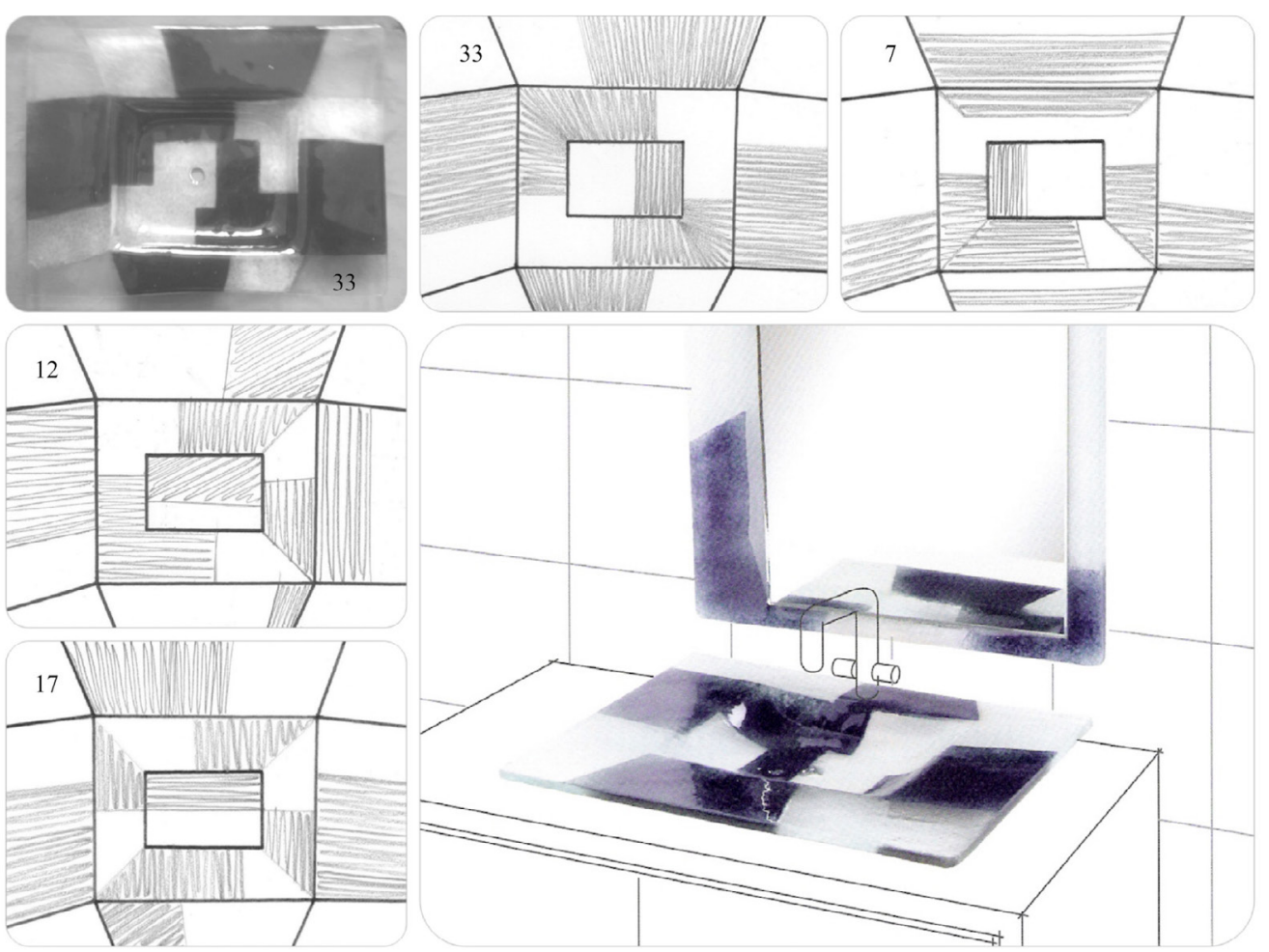

Fig. 5. CJC, AGG. Solución ornamental de proceso secuencial para diseño de Lavabo realizado por Cayetano Cruz para Andrea Seimandi 


\section{Hacia una Artesanía Asistida por Computador}

Nuestro planteamiento se esclarece desde la perspectiva descrita en el argumentario, verso la creatividad arte - artesanía - computación - tecnología. Por lo tanto, se expresa el interés por elaborar un ontológico que se adecue a los escenarios del arte y la artesanía, y que permita elaborar soluciones en las que la computación y la tecnología estén en sintonía con el pensamiento y los descriptores de la artesanía. De este modo, sería posible establecer relaciones de similitud entre el pensamiento creativo hacedor y el desarrollo tecnológico del pensamiento computacional; lo que se enmarca en la posibilidad de elaborar propuestas y soluciones hacia la "Artesanía Asistida por Computador".

En primera instancia, parecería que la artesanía y la tecnología pudieran estar en polos opuestos; $s i n$ embargo, la evolución tecnológica y de la inteligencia artificial son cada vez más cercanas y adaptada a las necesidades del usuario. Esto esclarece un mundo de posibilidades para la innovación en la artesanía; desde la ideación de productos mediante el uso de software adecuados a la manera de hacer del artesano, a la creación de productos generados desde el pensamiento de la computadora que "arropen" a los productos artesanales, pasando por establecer un método secuencial que adiestre y posibilite la acción del diseño creativo. En este último sentido se están desarrollando productos, como embalajes, expositores, ..., que dan soporte a las peculiaridades artesanales; de tal manera que cada pieza única pueda contar con un mismo embalaje, por ejemplo, que se adapta a la variabilidad de la forma sujeta a unos mismos parámetros.

Algunas de las experiencias vinculadas al estado del arte, y sus líneas de investigación, avalan la capacidad e idoneidad para para dar respuesta a objetivos de nuestro escenario particular; indicados para dar soluciones a ontológicos y sus concreciones.

\section{Agradecimientos}

Los autores desean expresar su gratitud al Ministerio de Economía y Competitividad de España, cuya trasferencia de investigación es posible a través del proyecto TIN2017-85727-C4-4-P, Deep-Bio-Uex, y al proyecto IB18093 de la Consejería de Economía e Infraestructuras del Gobierno Regional de Extremadura, cofundado por Fondo Europeo de Desarrollo Regional, 'Una forma de construir Europa'. 